\title{
The Phase-Shifting Limit Cycles of the van der Pol Equation
}

\author{
Peter J. Melvin* \\ Center for Applied Mathematics, National Measurement Laboratory National Bureau of Standards, \\ Boulder, Colorado 80303
}

(August 24, 1978)

\begin{abstract}
The van der Pol limit cycles are generated at small amplitudes by the computer implementation of the Poincaré-Lindstedt method. The formal algebraic solution is accomplished by manipulations of Poisson series, and the FORTRAN programming of the inductive algorithm yields the phase-shifting limit cycles to graphical accuracy over the range $0 \leq \lambda \leq 1.5$. This improves upon the method of Deprit and Rom in two ways. First, because the formal solution is carried out by hand, an algebraic processor is not necessary. Second, the standard solutions which they generated are only valid for $0 \leq \lambda \leq 1.2$ whereas the phase-shifting limit cycles are still valid at $\lambda=1.5$; that is, they do not exhibit the Gibbs phenomenon even at $\lambda=1.5$.

Key words: Analytical solutions; differential equations; mathematical software; nonlinear oscillations.
\end{abstract}

\section{Introduction}

The computer generation of the Poincaré-Lindstedt solutions to nonlinear differential equations is reported by Deprit and Rom in [1], ${ }^{1}$ and the term Poisson series for combined power-Fourier series is coined in [2]. More recently the Poincaré-Lindstedt method has been reapplied to different forms of the nonlinear oscillator equation in [3], [4], and [5].

In this paper the lead of [6] is followed, and the limit cycles of the van der Pol equation are constructed at small amplitudes. In the method of Deprit and Rom, a machine language algebraic processor is used to manipulate the Poisson series. It is shown here that it is not necessary to use such involved software tools because these algebraic operations can be carried out by hand, and the resulting system of recursion relations can be implemented in a higher level language such as FORTRAN. The bonus for first explicitly developing the recurrence relations for the van der Pol equation is that the existence of the phase-shifting cycles becomes apparent, and they can be generated to a larger value of the parameter than the standard cycles.

\section{Solution by Poisson Series}

The van der Pol equation

$$
Y^{\prime \prime}(t)-\lambda\left(1-Y^{2}\right) Y^{\prime}+Y=0,
$$

has enjoyed considerable interest in the literature of applied mathematics because it has singular, periodic solutions called limit cycles. The approach of the general solution to the limit cycle can be seen in the results of Davis in [7]. The cycles have been computed for all values of the parameter by purely numerical methods by Urabe in [8] and Clenshaw in [9]. In fact the numerical production of cycles is so simple it is given as an excercise in [10].

A more difficult task is their analytical production. The method of Poincare-Lindstedt as applied to (1) is well known and can be found in Cesari [11]. As noted in [2] and [3], an advantage to the computer implementation of the Poincaré-Lindstedt method is the speed and accuracy with which the whole process can

\footnotetext{
* Present address: Martin-Marietta Aerospace, P.O. Box 179, Denver, Colorado 80201.
}

${ }^{1}$ Figures in brackets indicate the literature references at the end of the paper. 
be performed. That is, within the interval of convergence the numerical evaluation of the Poisson series by Horner's rule and Goertzel's algorithm, see Coffey and Deprit [14], yields numerical values very much more rapidly than through normal methods of numerical integration.

The method of [5] is extended to the van der Pol equation by the assumption that the limit cycles have the Poisson series expansion (1.3) in table 1 , where $i=(-1)^{1 / 2}$. The frequency of the cycle $\omega$ is defined by $\tau$

TABle 1. Definitions of Coefficients

$\begin{aligned} \omega & =\sum_{m=1}^{\infty} \lambda^{2 m-2} \omega_{m}, \quad(1.1) ; \quad \omega^{2}=\sum_{m=1}^{\infty} \lambda^{2 m-2} \Omega_{m}, \\ Y & =\sum_{j=-\infty}^{\infty} e^{i(2 j-1) \tau} \sum_{m=1}^{\infty} \lambda^{2 m-2}\left(R_{m, j}^{(1)}+i \lambda I_{m, j}^{(1)}\right), \\ Y^{2} & =\sum_{j=-\infty}^{\infty} e^{i(2 j-2) \tau} \sum_{m=1}^{\infty} \lambda^{2 m-2}\left(R_{m, j}^{(2)}+i \lambda I_{m+1, j}^{(2)}\right) \\ \lambda Y^{2} Y^{\prime} & =\sum_{j=-\infty}^{\infty} e^{i(2 j-1) \tau} \sum_{m=1}^{\infty} \lambda^{2 m-2}\left(R_{m, j}^{(3)}+i \lambda I_{m, j}^{(3)}\right)\end{aligned}$

$=\omega t$ where the $\tau$-period is $2 \pi$. The algorithm is simplified by expanding both $\omega$ and $\omega^{2}$ as given in (1.1) and (1.2). By standard series manipulations, the problem of integrating (1) is reduced to the inductive algorithm of table 2 . The indicial conditions on the algorithm are

$$
R_{1,1}^{(1)}=\omega_{1}=\Omega_{1}=1 .
$$

The reflection relations are also given in table 2; they are used to obtain real solutions and to limit storage of coefficients to positive values of $j$. At the end of each equation in table 2 the selection rules are given which each nonzero coefficient must satisfy for positive $j$. For indices outside of the range of the selection rules, the corresponding coefficient is zero.

In table 2 recursion relations (2.1) and (2.5) are derived by substitution of (1.3) in the left-hand side of (1.4) in table 1 and by equating coefficients of equal powers of $\lambda e^{i \tau}$. Recursion relations (2.2) and (2.6) are obtained by substitution of (1.1), (1.3), and (1.4) in the left-hand side of (1.5). Because the nonlinear term in (1) can be expressed algebraically as the Poisson series (1.5), which is of the same form as the solution series (1.3), it might be called the nonlinear closure property of Poisson series.

By substitution of (1.3) and (1.5) in (1), two solution relations are obtained as

$$
-(2 j-1)^{2} \sum_{k=1}^{m} \Omega_{m-k+1} R_{k, j}^{(1)}+(2 j-1) \sum_{k=1}^{m-1} \omega_{m-k} I_{k, j}^{(1)}+R_{m, j}^{(3)}+R_{m, j}^{(1)}=0,
$$

and

$$
-(2 j-1)^{2} \sum_{k=1}^{m} \Omega_{m-k+1} I_{k, j}^{(1)}+(2 j-1) \sum_{k=1}^{m} \omega_{m-k+1} R_{k, j}^{(1)}+I_{m, j}^{(3)}+I_{m, j}^{(1)}=0 .
$$

When $j \geq 2$, relation (3) is solved with the indicial conditions (2) as (2.9). When $j=1$, relation (3) gives by (2.4) the coefficients in the square of the frequency as (2.7). This is Lindstedt's method of casting out secular terms. For $j=1$, relation (3) leaves arbitrary the sequence of linear amplitudes $R_{m, 1}^{(1)}$.

When $j \geq 2$, relation (4) is solved by (2) as (2.3). When $j=1$, relation (4) leaves $I_{m, 1}^{(1)}$ arbitrary, but by (2.4) the sequence of linear amplitudes is determined by the algebraic equation 
Set $m=1$.

$$
\begin{array}{ll}
R_{m, j}^{(2)}=R_{m, 2-j}^{(2)}=\sum_{p=-\infty}^{\infty}\left[\sum_{k=1}^{m} R_{m-k+1, j-p}^{(1)} R_{k, p}^{(1)}-\sum_{k=1}^{m-1} I_{m-k, j-p}^{(1)} I_{k, p}^{(1)}\right], & 2 m \geq j \geq 1 . \\
I_{m, j}^{(3)}=-I_{m, 1-j}^{(3)}=\sum_{p=-\infty}^{\infty}(2 p-1)\left[\sum_{k=1}^{m} R_{m-k+1, j-p+1}^{(2)} \sum_{n=1}^{k} \omega_{k-n+1} R_{n, p}^{(1)}-\sum_{k=2}^{m} I_{m-k+2, j-p+1}^{(2)} \sum_{n=1}^{k-1} \omega_{k-n} I_{n, p}^{(1)}\right], & 2 m \geq j \geq 1 . \\
I_{m, j}^{(1)}=-I_{m, 1-j}^{(1)}=\left[I_{m, j}^{(3)}-(2 j-1) \sum_{k=1}^{m} \omega_{m-k+1} R_{k, j}^{(1)}-(2 j-1)^{2} \sum_{k=1}^{m-1} \Omega_{m-k+1} I_{k, j}^{(1)}\right] / 4 j(j-1), & 2 m \geq j \geq 2 . \\
I_{m, 1}^{(1)}=0 .
\end{array}
$$

The m-th approximation is complete, replace $m$ by $m+1$.

$$
\begin{aligned}
& I_{m, j}^{(2)}=-I_{m, 2-j}^{(2)}=\sum_{p=-\infty}^{\infty} \sum_{k=1}^{m-1}\left(R_{m-k, j-p}^{(1)} I_{k, p}^{(1)}+I_{m-k, j-p}^{(1)} R_{k, p}^{(1)}\right), \\
& R_{m, j}^{(3)}=R_{m, 1-j}^{(3)}=-\sum_{p=-\infty}^{\infty}(2 p-1)\left[\sum_{k=2}^{m} R_{m-k+1, j-p+1}^{(2)} \sum_{n=1}^{k-1} \omega_{k-n} I_{n, p}^{(1)}+\sum_{k=1}^{m-1} I_{m-k+1, j-p+1}^{(2)} \sum_{n=1}^{k} \omega_{k-n+1} R_{n, p}^{(1)}\right], \\
& \Omega_{m}=R_{m, 1}^{(3)}-\sum_{k=2}^{m-1} \Omega_{m-k+1} R_{k, 1}^{(1)} . \\
& \omega_{m}=\frac{k}{2}\left[\Omega_{m}-\sum_{k=2}^{m-1} \omega_{m-k+1} \omega_{k}\right] . \\
& R_{m, j}^{(1)}=R_{m, 1-j}^{(1)}=[2.5) \\
& \left.R_{m, j}^{(3)}+(2 j-1) \sum_{k=2}^{m-1} \omega_{m-k} I_{k, j}^{(1)}-(2 j-1)^{2} \sum_{k=2}^{m-1} \Omega_{m-k+1} R_{k, j}^{(1)}\right] / 4 j(j-1)
\end{aligned}
$$

Set $R_{m, 1}^{(1)}=0$ and compute $(2.1)$ and $(2.2)$, then compute

$$
R_{m, 1}^{(1)}=\frac{1}{2}\left[\sum_{k=1}^{m-1} \omega_{m-k+1} R_{k, 1}^{(1)}-I_{m, 1}^{(3)}\right] .
$$

$$
I_{m, 1}^{(3)}-\sum_{k=1}^{m} \omega_{m-k+1} R R_{k, 1}^{(1)}=0
$$

The coefficients $R_{m, 1}^{(1)}$ occur explicitly in the second term in (5) and implicitly in the first. To make this dependence explicit, (2.1) is rewritten as

$$
R_{m, 1}^{(2)}=4 R_{m, 1}^{(1)},+\bar{R}_{m, 1}^{(2)} \text {, and } R_{m, 2}^{(2)}=2 R_{m, 1}^{(1)}+\bar{R}_{m, 2}^{(2)}
$$

where the remainder $\bar{R}_{m, j}^{(2)}$ is independent of $R_{m, 1}^{(1)}$. Relation (2.2) is rewritten by (6) as

$$
I_{m, 1}^{(3)}=3 R_{m, 1}^{(1)}+\bar{I}_{m, 1}^{(3)}
$$


where the remainder $\bar{I}_{m, 1}^{(3)}$ is also independent of $R_{m, 1}^{(1)}$. The remainder terms are easily found in a computer program by setting $R_{m, 1}^{(1)}$ to zero and by then executing the subroutines for (2.1) and (2.2). Substitution of (7) in (5) gives (2.10) upon solution for $R_{m, 1}^{(1)}$. Thus, the dissipation terms in the van der Pol equation determine the sequence of linear amplitudes.

\section{Standard and Phase-Shifting Cycles}

When $j=1$, relation (4) leaves arbitrary the phase amplitudes $I_{m, 1}^{(1)}$ and unlike $R_{m, 1}^{(1)}$ their values are not determined by the van der Pol equation itself. This harks back to the fact that (1) is autonomous. In [6], [7], [8], and [9] the phase of the cycle is determined by imposing the initial condition

$$
Y^{\prime}(0)=0,
$$

for all values of $\lambda$. That is, the phase of the cycles is adjusted so that the initial amplitude is a maximum. To fulfill this condition, it is found from (1.3) that the phase amplitudes must satisfy

$$
I_{m, 1}^{(1)}=-\sum_{j=2}^{2 m}(2 j-1) I_{m, j}^{(1)} .
$$

Because so many investigators impose the condition (8), here the resulting solutions are called the standard cycles.

The arbitrary sequence $I_{m, 1}^{(1)}$ can be chosen, however, in other ways. In particular the simple choice (2.4) can be made. When $I_{m, 1}^{(1)}$ is not chosen to satisfy (9), then the condition (8) no longer holds. The resulting cycles are said to be phase-shifting, and table 2 is arranged to generate coefficients for phase-shifting cycles. The algorithm for the standard cycles is obtained by replacement of (2.4) by (9), by replacement of (2.7) by

$$
\Omega_{m}=R_{m, 1}^{(3)}-\sum_{k=2}^{m-1} \Omega_{m-k+1} R_{k, 1}^{(1)}+\sum_{k=1}^{m-1} \omega_{m-k} I_{k, 1}^{(1)}
$$

and by replacement of $(2.10)$ by

$$
R_{m, 1}^{(1)}=1 / 2\left[\sum_{k=1}^{m-1}\left(\omega_{m-k+1} R_{k, 1}^{(1)}+\Omega_{m-k+1} I_{k, 1}^{(1)}\right)-\bar{I}_{m, 1}^{(3)}\right] .
$$

These replacements require the algorithm for the standard solutions to have more arithmetic operations than for the phase-shifting solutions. It is probably for this reason that the phase-shifting cycles can be produced to larger values of the parameter than the standard cycles.

\section{Comparison of Results}

To compare results with previous investigators, the standard cycles were generated. The amplitude of the standard solutions is

$$
a(\lambda) \equiv Y(\lambda, 0)=\sum_{m=1}^{\infty} a_{m} \lambda^{2 m-2},
$$

where the amplitude coefficients are computed from the linear amplitudes by

$$
a_{m}=2 \sum_{j=1}^{2 m-1} R_{m, j}^{(1)}
$$

Other than by direct comparison, there is no simple way to determine how the various sources of error affect the final results. To study the propagation of error, the program was executed on an IBM $360 / 75$ at the University of Illinois at Urbana-Champaign in single precision which gives six to seven decimal digits. The 
first column of table 3 contains the results of Deprit and Rom who used double precision on an IBM 7094, and the second column contains results of this investigation. The coefficients in the two columns show perfect agreement with each other and with the rational fractions found by hand by Clenshaw in [9] only to order $m$ $=3$. At order $m=4$ there is a disadvantageous subtraction, and there is only two decimal agreement. That there is such a disagreement is expected since Deprit and Rom used so many significant digits. What is surprising is that the disagreement occurs at such a low order. The fourth order is beyond my ability for hand computation, and it would be interesting to see the method of [4] applied to (1) to give at least the $m=4$ coefficients as rational fractions.

TABLE 3. Amplitude and Frequency Coefficients

\begin{tabular}{|c|c|c|c|c|}
\hline$m$ & $\mathrm{a}_{\mathrm{m}}$ (Deprit \& Rom) & $a_{m}$ & $\omega_{m}$ & $\mathrm{r}_{m}$ \\
\hline 1 & 2 & 2 & 1 & 1 \\
\hline 2 & $0.010 \quad 416 \quad 666 \quad 667$ & $1.04167 \mathrm{E}-2$ & $-6.25000 \mathrm{E}-2$ & 2.52 \\
\hline 3 & $-0.001 \quad 868 \quad 127893$ & $-1.86813 \mathrm{E}-3$ & $5.53385 \mathrm{E}-3$ & 2.83 \\
\hline 4 & $\begin{array}{llll}0.000 & 018 & 294 & 203\end{array}$ & $1.83739 \mathrm{E}-5$ & $3.95598 \mathrm{E}-5$ & 4.26 \\
\hline 5 & $\begin{array}{lllll}0.000 & 062 & 520 & 749\end{array}$ & $6.25358 \mathrm{E}-6$ & $-1.33220 \mathrm{E}-4$ & 2.70 \\
\hline 6 & $-0.000 \quad 007 \quad 989633$ & $-7.98043 \mathrm{E}-6$ & $1.22798 \mathrm{E}-5$ & 2.80 \\
\hline 7 & $-0.000 \quad 002 \quad 189064$ & $-2.22516 \mathrm{E}-6$ & $4.52331 \mathrm{E}-6$ & 2.58 \\
\hline 8 & $0.000 \quad 000 \quad 678 \quad 892$ & $6.76593 \mathrm{E}-7$ & $-1.09645 \mathrm{E}-6$ & 2.50 \\
\hline 9 & $0.000 \quad 000 \quad 048985$ & 4.81233E-8 & $-1.25366 \mathrm{E}-7$ & 2.55 \\
\hline 10 & $-0.000 \quad 000 \quad 046 \quad 470$ & $-4.29809 \mathrm{E}-8$ & $7.70256 \mathrm{E}-8$ & 2.37 \\
\hline 11 & $0.000 \quad 000 \quad 002 \quad 256$ & $2.20042 \mathrm{E}-9$ & $-1.04284 \mathrm{E}-9$ & 2.68 \\
\hline 12 & $\begin{array}{llll}0.000 & 000 & 002 & 732\end{array}$ & $2.90587 \mathrm{E}-9$ & $-4.70514 \mathrm{E}-9$ & 2.30 \\
\hline 13 & $-0.000 \quad 000 \quad 000 \quad 465$ & $-8.84975 \mathrm{E}-10$ & $6.03579 \mathrm{E}-10$ & 2.34 \\
\hline 14 & $-0.000 \quad 000 \quad 000 \quad 130$ & $-1.49888 \mathrm{E}-10$ & $2.41538 \mathrm{E}-10$ & 2.27 \\
\hline 15 & $\begin{array}{llll}0.000 & 000 & 000 & 047\end{array}$ & $3.97400 \mathrm{E}-11$ & $-6.86656 \mathrm{E}-11$ & 2.24 \\
\hline 16 & $0.000 \quad 000 \quad 000 \quad 042$ & $5.08794 \mathrm{E}-11$ & $-8.57589 \mathrm{E}-12$ & 2.28 \\
\hline 17 & & $-5.45275 \mathrm{E}-12$ & $5.78845 \mathrm{E}-12$ & 2.19 \\
\hline 18 & & & $-7.64649 \mathrm{E}-14$ & 2.37 \\
\hline 19 & & & $-4.04249 \mathrm{E}-13$ & 2.16 \\
\hline 20 & & & $5.35501 \mathrm{E}-14$ & 2.19 \\
\hline 21 & & & $2.30394 \mathrm{E}-14$ & 2.15 \\
\hline 22 & & & $-6.68763 \mathrm{E}-15$ & 2.14 \\
\hline
\end{tabular}

The agreement improves slightly at $m=5$ and 6 , but it worsens as higher orders are reached. On the other hand, the Poincaré-Lindstedt method is numerically stable because even at order 15 the amplitude coefficients disagree with a relative error of only 15 percent. Although Deprit and Rom report numerical values to order 16, there is an additional unknown correction in their last coefficients. Evidently they output their coefficients after (2.9) but before evaluating (2.10). A better output point is after (2.4) as then the order $m$ is completed in (11) and (12).

The disagreement in the amplitude coefficients is not significant, however, because it is the values of $a(\lambda)$, $\omega(\lambda), Y(\lambda, \tau)$ and $Y^{\prime}(t)$ which are more important. The values of the amplitude and frequency are given in table 4. The purely numerical results of [6] or [9] are listed in the first and third columns. The IBM single precision evaluation in the second column shows perfect agreement for $0 \leq \lambda \leq 1.5$ and is in relative error of -0.02 percent at $\lambda=1.75$. The value obtained by Deprit and Rom for $a(1.75)$ is slightly better with a relative error of -0.004 percent.

Even though Deprit and Rom used twice as many digits in their implementation of the Poincaré-Lindstedt method, they were unable to produce limit cycles without the Gibbs phenomenon beyond $\lambda=1.2$. The reason for this is apparent from figures 1.1 and 1.2. The imposition of the standard condition (8) apparently causes the early onset of the uncontrolled oscillation. In figure 1.1 the standard cycles are plotted for order 17 for $\lambda$ $=0,0.5,1.0$, and 1.5 as obtained in IBM single precision.

Figures $1.1,1.2$, and 1.3 overlap so that figure 1.1 refers to the first two families of curves. Figure 1.2 refers to the second pair of families. The top family of curves are $Y(\lambda, \tau)$ for $\lambda=0,0.5,1.0$, and 1.5 ; and the 


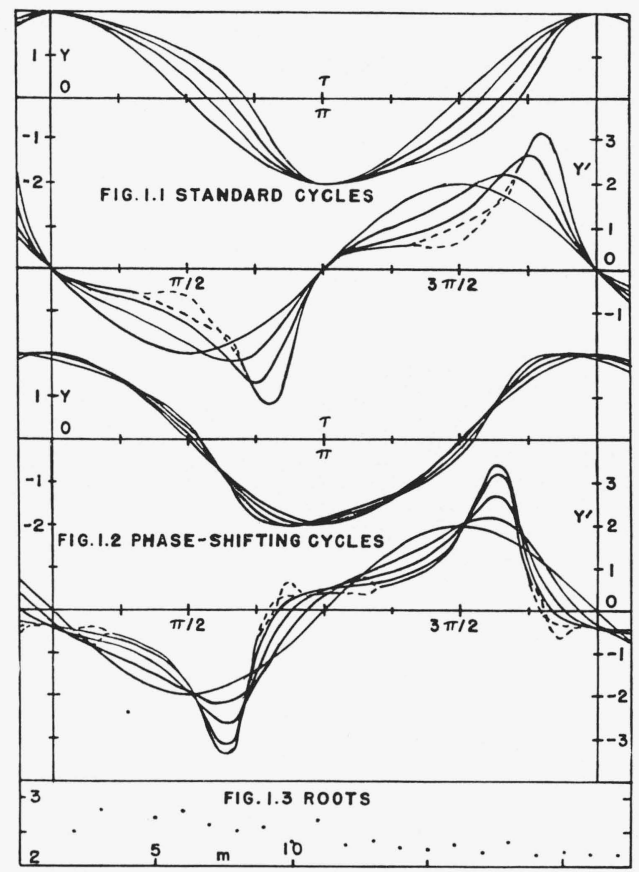

FigURE 1. 1.1 shows standard cycles; 1.2 shows phaseshifting cycles; 1.3 shows roots.

second family of curves are $Y^{\prime}(t)$ for the same values of $\lambda$. The curves of figure 1.1 represent the standard cycles computed by the method of this paper. The dashed lines for $Y^{\prime}(t)$ in figure 1.1 are obtained from the $m=16$ - and 17-th order polynomials and clearly exhibit the Gibbs phenomenon at $\lambda=1.5$. The onset of the Gibbs phenomenon occurred in Deprit and Rom's results at $\lambda=1.35$.

In figure 1.2, the first family of curves are the phase-shifting limit cycle solutions $Y(\lambda, \tau)$ for $\lambda=0,0.5$, 1.0, and 1.5. The second family in figure 1.2 is $Y^{\prime}(t)$ for $\lambda=0,0.5,1.0,1.5$, and 1.75. Thus, it is possible to generate the phase-shifting limit cycles of the van der Pol equation without the Gibbs phenomenon at $\lambda=$ 1.5. The Gibbs phenomenon is, however, exhibited at $\lambda=1.75$ in the phase-shifting cycles as can be seen in figure 1.2 where the $m=21$-st and 22-nd order polynomials are plotted as dashed curves.

\section{The Phase-Shifting Cycles}

When the algorithm of table 2 was executed in IBM single precision, it was found that unlike the standard solutions there is a negligible amount of the Gibbs phenomenon in the phase-shifting solutions at $\lambda=1.5$ for $m=15$. The program for the phase-shifting cycles was again run on a CYBER 175 where the program achieved the 22nd order in 130 seconds. The 60 bit, single precision word of the CYBER accumulator gives 14 decimal digits of significance. All of the frequency coefficients are listed to six decimals in the third column of table 3 , and the solution coefficients are given to the 6th order in tables 5 and 6 . In the fourth column of table 4 values of the frequency are given, and there is perfect agreement with the third column for $0 \leq \lambda \leq 1.5$. The 22 nd order phase-shifting cycles are plotted in figure 1.2. In addition, $Y^{\prime}(t)$ is plotted for $\lambda=1.75$ to show the Gibbs phenomenon at orders 21 and 22 as dashed lines. Thus, by not imposing the constraint (8), the Poincaré-Lindstedt method of constructing the limit cycles of the van der Pol equation yields at least graphically accurate results over the interval $0 \leq \lambda \leq 1.5$.

\section{Convergence of the Series}

Although the Poisson series solution to (1) is shown rigorously to converge at very small values of $\lambda$ by Hale in [12], it is not obvious for how large a value of the parameter the series continues to converge. Because the 
TABLE 4. Amplitude and Frequency

\begin{tabular}{c|c|c|c|c|c}
\hline \hline$\lambda$ & $\mathrm{a}(\lambda),[6,9]$ & $\mathrm{a}(\lambda), m=17$ & $\omega(\lambda),[6,9]$ & $\omega(\lambda), m=22$ & $\omega(\lambda), m=3$ \\
\hline 0.5 & 2.0024879 & 2.002487 & 0.9847210 & 0.984721 & 0.984721 \\
1.0 & 2.0086199 & 2.008619 & 0.9429558 & 0.942956 & 0.943034 \\
1.5 & 2.0152265 & 2.015227 & 0.8854079 & 0.885408 & 0.887390 \\
1.75 & 2.0178601 & 2.018298 & 0.8544505 & 0.854436 & 0.860495 \\
\hline
\end{tabular}

character of the solution to the linearized form of (1) changes at $\lambda=2$ from underdamped to overdamped, it is expected that $\lambda=2$ is the radius of convergence of the series in table 1 . When series solutions are generated on a computer, the radius of convergence is not usually known beforehand; and it is helpful if the program can determine the range of validity of the series. The root test used in [13] is more reliable than the ratio test used in [5]. It can be seen in the last column of table 3 and in figure 1.3 that the roots

$$
r_{m}=\left|\omega_{m}\right|^{-1 /(2 m-2)}
$$

appear to have the lower bound of 2 .

By comparing the accuracy of the approximating polynomials at order $m$ with the behavior of the roots, it is found that the most accurate polynomials are those for the order just before a large increase in the root. For example, in table 3 there is a jump in the root at $m=4$; and it is seen in the last column of table 4 that a good approximation to the frequency is obtained at the $3 \mathrm{rd}$ order.

\section{Lower Order Approximations}

The real form of the phase-shifting cycles is found from (1.3), the reflection and selection rules as

$$
Y(\lambda, \tau)=2 \sum_{m=1}^{\infty} \lambda^{2 m-2}\left[\sum_{j=1}^{2 m-1} R_{m, j}^{(1)} \cos (2 j-1) \tau-\lambda \sum_{j=2}^{2 m} I_{m, j}^{(1)} \sin (2 j-1) \tau\right] .
$$

By the chain rule the derivative is found to be

$$
Y^{\prime}(t)=-2 \omega \sum_{m=1}^{\infty} \lambda^{2 m-2}\left[\sum_{j=1}^{2 m-1}(2 j-1) R_{m, j}^{(1)} \sin (2 j-1) \tau+\lambda \sum_{j=2}^{2 m}(2 j-1) I_{m, j}^{(1)} \cos (2 j-1) \tau\right] .
$$

The greatest problem in the numerical evaluation of the limit cycles is the Gibbs phenomenon in the derivative (12). To see what order is required to obtain graphical accuracy for a given $\lambda$, a TUTOR language evaluation of (1.1), (11), and (12) was used on the PLATO IV system at the University of Illinois. In figure 2 the phaseshifting cycles are plotted for $\lambda=0,0.25,0.5,0.75,1.0,1.25$, and 1.5 which are obtained at orders 1,3 , $3,3,6,9$, and 15 . The PLATO storage is rather limited, but it could accommodate the 510 solution coefficients necessary for order 15 .

Because the total storage of table 2 is only 7750 coefficients to order 25 , it is perhaps not necessary to use a large computer to evaluate the algorithm. Since table 2 was coded in FORTRAN, it is probably possible to code it in the BASIC language of minicomputers. Although storage requirements are not prohibitive, computer time is a limitation. The execution time for each order appears to grow exponentially. For example, it took 3 minutes of IBM 360/75 time to reach order 15, but an additional two orders to $m=17$ required two minutes more.

In tables 5 and 6 , the real and imaginary solution coefficients are given to six figures to the 6th order. There are sufficient coefficients in these tables and table 3 to construct the solutions (1.1), (11), and (12) on a programmable calculator to graphical accuracy over the interval $0 \leq \lambda \leq 1.0$.

This investigation of the van der Pol equation was originally undertaken to gain understanding of the limit cycle phenomena. The problem of the limit cycle behavior of periodic variable stars is reported in [15]. 


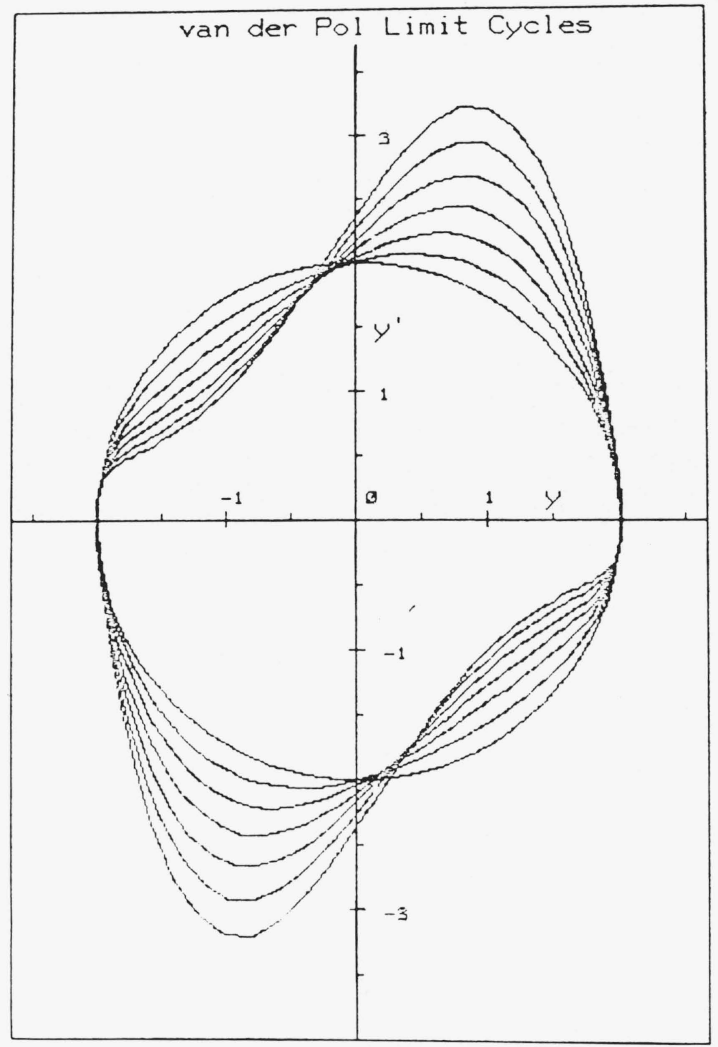

Figure 2. Plato display; van der Pol Limit Cycles.

Table 5. Real, Phase-Shifting Solution Coefficients $R_{m, j}^{(1)}$

\begin{tabular}{c|c|r|r|r|r}
\hline \hline$m / j$ & $1,6,11$ & \multicolumn{1}{|c|}{2,7} & \multicolumn{1}{c}{3,8} & \multicolumn{1}{c}{4,9} & \multicolumn{1}{c}{5,10} \\
\hline 1 & 1.0 & & & & \\
2 & $7.81250 \mathrm{E}-3$ & $-4.68750 \mathrm{E}-2$ & $-2.60417 \mathrm{E}-2$ & & \\
3 & $-2.33968 \mathrm{E}-4$ & $4.10970 \mathrm{E}-3$ & $8.43189 \mathrm{E}-3$ & $6.23463 \mathrm{E}-3$ & $1.48926 \mathrm{E}-3$ \\
4 & $-1.51937 \mathrm{E}-4$ & $4.24933 \mathrm{E}-4$ & $-6.45271 \mathrm{E}-4$ & $-1.71476 \mathrm{E}-3$ & $-1.49991 \mathrm{E}-3$ \\
& $-6.17692 \mathrm{E}-4$ & $-9.62417 \mathrm{E}-5$ & & & \\
5 & $2.42362 \mathrm{E}-5$ & $-1.71589 \mathrm{E}-4$ & $-1.90775 \mathrm{E}-4$ & $5.73677 \mathrm{E}-5$ & $3.38734 \mathrm{E}-4$ \\
& $3.62885 \mathrm{E}-4$ & $1.97997 \mathrm{E}-4$ & $5.64688 \mathrm{E}-5$ & $6.55160 \mathrm{E}-6$ & \\
6 & $3.98953 \mathrm{E}-6$ & $9.46432 \mathrm{E}-7$ & $4.82361 \mathrm{E}-5$ & $6.52477 \mathrm{E}-5$ & $1.05810 \mathrm{E}-5$ \\
& $-6.21963 \mathrm{E}-5$ & $-8.53587 \mathrm{E}-5$ & $-5.77692 \mathrm{E}-5$ & $-2.27698 \mathrm{E}-5$ & $-4.96343 \mathrm{E}-6$ \\
& $-4.59207 \mathrm{E}-7$ & & & & \\
\hline
\end{tabular}

TABle 6. Imaginary, Phase-Shifting Solution Coefficients $I_{m, j}^{(1)}$

\begin{tabular}{c|c|r|r|r|r}
\hline \hline$m / j$ & \multicolumn{1}{|c|}{$2,7,12$} & \multicolumn{1}{|c|}{3,8} & \multicolumn{1}{|c}{4,9} & \multicolumn{1}{|c}{6,10} & \\
\hline 1 & 0.125 & & & & \\
2 & $-1.46484 \mathrm{E}-2$ & $-1.84462 \mathrm{E}-2$ & $-6.07639 \mathrm{E}-3$ & & \\
3 & $3.30183 \mathrm{E}-4$ & $3.04987 \mathrm{E}-3$ & $3.76636 \mathrm{E}-3$ & $1.99198 \mathrm{E}-3$ & $3.75231 \mathrm{E}-4$ \\
4 & $3.33934 \mathrm{E}-4$ & $1.02407 \mathrm{E}-4$ & $-5.44143 \mathrm{E}-4$ & $-8.28345 \mathrm{E}-4$ & $-5.57686 \mathrm{E}-4$ \\
& $-1.87977 \mathrm{E}-4$ & $-2.49947 \mathrm{E}-5$ & & & \\
5 & $-4.74168 \mathrm{E}-5$ & $-1.22377 \mathrm{E}-4$ & $-6.87602 \mathrm{E}-5$ & $8.32629 \mathrm{E}-5$ & $1.79145 \mathrm{E}-4$ \\
& $1.48482 \mathrm{E}-4$ & $6.79942 \mathrm{E}-5$ & $1.68027 \mathrm{E}-5$ & $1.72960 \mathrm{E}-6$ & \\
6 & $-9.60169 \mathrm{E}-6$ & $9.72957 \mathrm{E}-6$ & $3.40962 \mathrm{E}-5$ & $2.76165 \mathrm{E}-5$ & $-7.88881 \mathrm{E}-6$ \\
& $-3.68516 \mathrm{E}-5$ & $-3.77886 \mathrm{E}-5$ & $-2.16231 \mathrm{E}-5$ & $-7.47641 \mathrm{E}-6$ & $-1.45772 \mathrm{E}-6$ \\
& $-1.22484 \mathrm{E}-7$ & & & & \\
\hline
\end{tabular}


The author wishes to thank Dr. André Deprit for several useful discussions and for the encouragement to complete this paper. Mr. Alec Himwich of the Computer-Based Educational Research Laboratory helped with the intricacies of the PLATO programming. Computer time was provided by the Office of the Dean of Liberal Arts and Sciences and the Department of Mathematics of the University of Illinois at Urbana-Champaign.

\section{REFERENCES}

[1] Deprit, A., and Rom, A., Poincaré's Continuation by Computers, AIAA Journal, 6, 7, 1234-1239 (1968).

[2] Deprit, A., and Rom, A., Lindstedt's Series on a Computer, Astron. Journal, 73, 3, 210-213 (1968).

[3] Helleman, R. H. G., and Montroll, E. W., On a Nonlinear Perturbation Theory without Secular Terms, Physica, 74, 22-74 (1974).

[4] Alfriend, K. T., and Pickard, H. M., Solution of the Perturbed Harmonic Oscillator by Computer, Naval Research Laboratory Memorandum Report, №. 3513 (1977).

[5] Melvin, P. J., On the Construction of the Poincaré-Lindstedt Solutions: The Nonlinear Oscillator Equation, SIAM J. Appl. Math., 33, 1, 161-194 (1977).

[6] Deprit, A., and Rom, A., Asymptotic Representation of the Cycle of van der Pol's Equation for Small Damping Coefficients, Z. Angew. Math. Phys., 18, 736-747 (1967).

[7] Davis, H. T., Introduction to Nonlinear Differential and Integral Equations (Dover, New York, 1962).

[8] Urabe, M., Numerical Study of Periodic Solutions of the van der Pol Equation, in Nonlinear Differential Equations and Nonlinear Mechanics, J. P. LaSalle and S. Lefshetz, Eds. (Academic Press, New York, 1963).

[9] Clenshaw, C. W. The Solution of van der Pol's Equation in Chebyshev Series, in Numerical Solutions of Nonlinear Differential Equations, D. Greenspan, Ed. (John Wiley, New York, 1966), 55-63.

[10] Shampine, L. F., and Allen, R. C., Numerical Computing: An Introduction (W. F. Saunders Company, Philadelphia, 1973).

[11] Cesari, L., Asymptotic Behavior and Stability Problems in Ordinary Differential Equations, Second Edition (Academic Press, New York, 1963).

[12] Hale, J. K., Oscillations in Nonlinear Systems (McGraw-Hill, New York, 1963).

[13] Barton, D., Willers, I. M., and Zahar, R. V. M., Taylor Series Methods for Ordinary Differential Equations - An Evaluation, in Mathematical Software, J. R. Rice, Ed. (Academic Press, New York, 1971), 369-390.

[14] Coffey, S., and Deprit, A., Fast Evaluation of Fourier Series, AIAA/AAS Astrodynamics Conference, Palo Alto, California, August 7-9, 1978, Paper No. 78-1384, pp. 1-6 (American Institute of Aeronautics and Astronautics, 1290 Avenue of the Americas, New York, N. Y. 10019, 1978).

[15] Melvin, P. J., An Analytical Theory of Periodic Variable Stars, to appear in Quart. of Appl. Math. 